\title{
Acute Interstitial Pneumonia Induced by ONO-1078 (pranlukast), a Leukotriene Receptor Antagonist
}

\author{
Naotsugu TaKahashi ${ }^{* * *}$, Tomoaki Iwanaga**, Hisamichi Aizawa***, Hiroshi Koto*, Kentaroh Watanabe****, \\ Reiko KishikawA**, Togo IKedA**, Shunsuke ShoJ ${ }^{* *}$, Sankei NiShIMA** and Nobuyuki HarA*
}

\begin{abstract}
A 62-year-old woman treated with pranlukast for 2 months developed interstitial pneumonitis with a high fever. A lymphocyte stimulation test was reactive to pranlukast. Her clinical symptoms improved with discontinuation of pranlukast and administration of systemic corticosteroid. To our knowledge, this is the first reported case of drug-induced lung disease involving a leukotriene. The steps that can be taken to promptly reach a diagnosis and to successfully treat this life-threatening condition are described.
\end{abstract}

(Internal Medicine 40: 791-794, 2001)

Key words: leukotriene receptor antagonist, pranlukast, zafirlukast, drug-induced interstitial pneumonia

\section{Introduction}

Pranlukast, a leukotriene receptor antagonist, was recently approved to control persistent asthma $(1,2)$. Only a few side effects have been reported in association with leukotriene receptor antagonists including pranlukast, zafirlukast and montelukast; these include Churg-Strauss syndrome (3), pulmonary infiltrates with eosinophilia after corticosteroid withdrawal (4) and ulcerative colitis after corticosteroid withdrawal (5). Here, we describe here a case of interstitial pneumonitis likely induced by pranlukast.

\section{Case Report}

A 62-year-old woman with a 10-year history of severe persistent asthma was referred to our hospital for dyspnea and further evaluation of steroid- (prednisolone, $5 \mathrm{mg}$ ) dependent asthma. She had been taking prednisolone during the prior 12 months. Physical examination revealed afebrile tachypnea. Her temperature was $36.3^{\circ} \mathrm{C}$, and she showed remarkable cyano- sis. Auscultation revealed continuous wheeze throughout both lungs. No abnormality was found in the peripheral regions, and neurological findings were normal.

Her white blood cell (WBC) count was $6,800 \mathrm{~mm}^{-3}$; she had 3.2\% eosinophils, and an IgE level of 34 units, she was negative for antibodies to aspergillus. Her erythrocyte sedimentation rate was $25 \mathrm{~mm} / \mathrm{h}$, and her C-reactive protein level was less than $0.3 \mathrm{mg} / \mathrm{dl}$; lactate dehydrogenase was normal at 417 IU/l (normal, 230-480 IU). Other serum and serologic measures were within normal limits. Chest radiograph was normal. Blood gas analysis showed an oxygen tension $\left(\mathrm{PaO}_{2}\right)$ of $58 \mathrm{mmHg}$ and carbon dioxide tension $\left(\mathrm{PaCO}_{2}\right)$ of $44 \mathrm{mmHg}$, while the patient was breathing room air.

For the treatment of the bronchial asthma, we started prednisolone $(80 \mathrm{mg} /$ day $)$ and pranlukast $(450 \mathrm{mg} / \mathrm{day})$. On day 20 after the start of treatment, the patient's temperature had risen to $37.5^{\circ} \mathrm{C}$. Auscultation again revealed severe wheeze throughout both lungs. Consequently, we increased the dose of prednisolone to $100 \mathrm{mg} / \mathrm{day}$. On day 25 , her WBC count was elevated to $11,840 \mathrm{~mm}^{-3}$ with no eosinophilia. Her erythrocyte sedimentation rate was $1 \mathrm{~mm} / \mathrm{h}$, and her C-reactive protein level remained at less than $0.3 \mathrm{mg} / \mathrm{dl}$. Lactate dehydrogenase was elevated to $716 \mathrm{IU} / l$. Other serum and serologic measures were within normal limits, and a chest radiograph was normal. Her $\mathrm{PaO}_{2}$ had improved to $84 \mathrm{mmHg}$ and her $\mathrm{PaCO}_{2}$ improved to $39 \mathrm{mmHg}$ while she was breathing room air. By day 35, the patient's general condition was so improved that we stopped administration of prednisolone and continued only with pranlukast. The patient's febrile condition continued, however, with a temperature of $38^{\circ} \mathrm{C}$. On day 40 , auscultation revealed late inspiratory fine crackles in both lower lung fields, and both chest radiography and computed tomography revealed interstitial bibasilar pneumonitis (Fig. 2). Blood gas analyses showed hypoxemia with a $\mathrm{PaO}_{2}$ of $66 \mathrm{mmHg}$ and $\mathrm{PaCO}_{2}$ of $38 \mathrm{mmHg}$ while the patient was breathing $2 \mathrm{l} / \mathrm{min} \mathrm{O}_{2}$ through a nasal cannula. Bronchoalveolar lavage (BAL) was not done because of severe hypoxemia and the possibility of pulmonary edema (6). Transbronchial lung biopsy (TBLB) revealed thickening of alveolar septa infiltrated by lymphocytes and the presence of

From *Research Institute for Diseases of the Chest, Faculty of Medicine, Kyushu University, Fukuoka, **National Minami-Fukuoka Hospital, Fukuoka, ***National Fukuoka-Higashi Hospital, Fukuoka and ****Respiratory Medicine, School of Medicine, Fukuoka University, Fukuoka 
Prednisolone

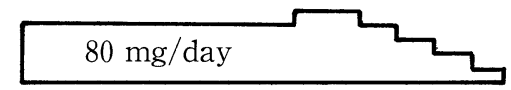

Pranlukast

$450 \mathrm{mg} /$ day

Fever

Wheeze

Fine crackle
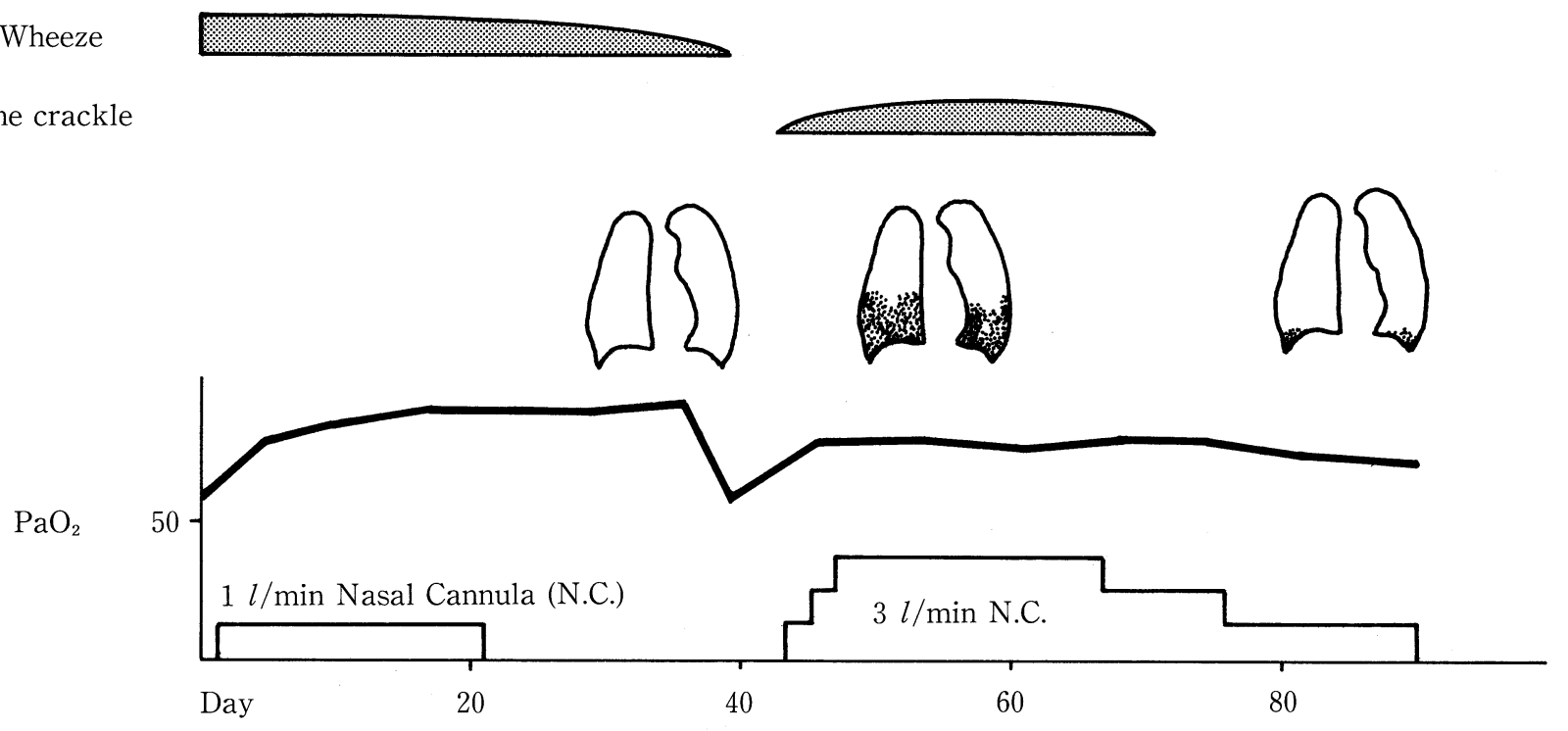

Figure 1. Clinical course from admission to improvement of interstitial pneumonitis.
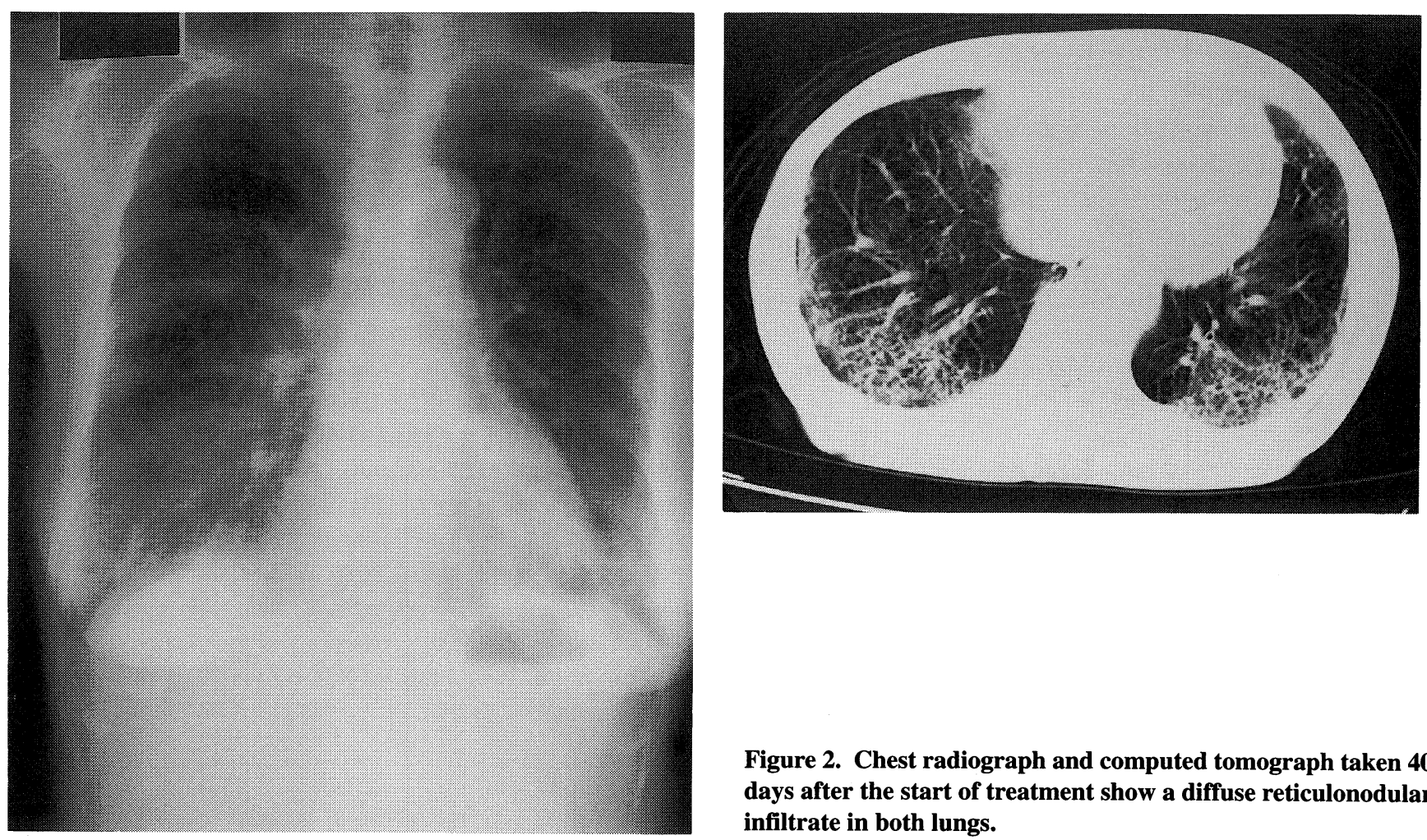

Figure 2. Chest radiograph and computed tomograph taken 40 days after the start of treatment show a diffuse reticulonodular infiltrate in both lungs. 


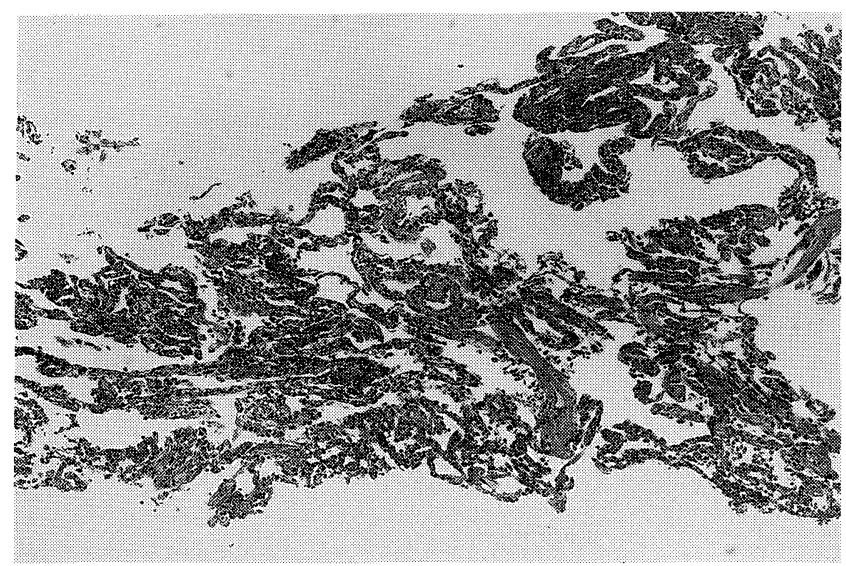

A

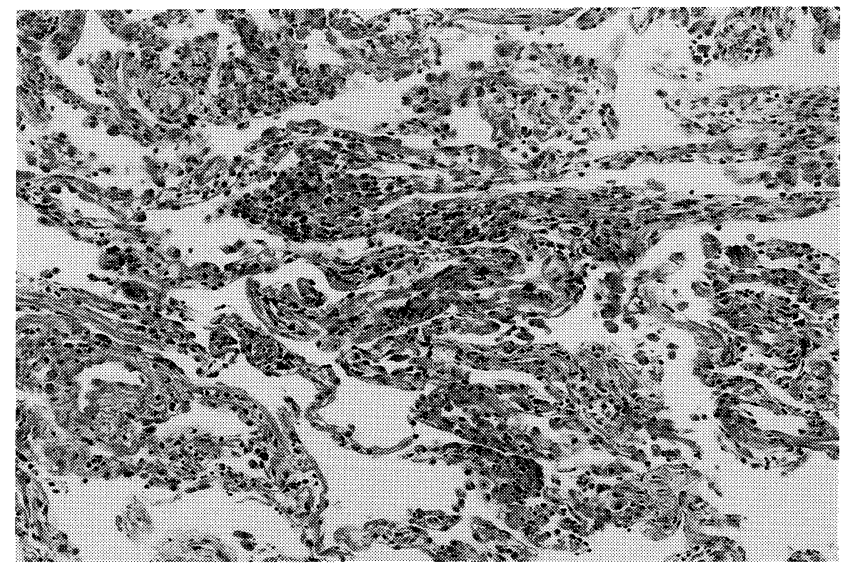

B

Figure 3. A) TBLB specimen shows that alveolar septa are thickened with lymphocytic infiltration: HE $\times 39.6$ (original magnification). B) Clusters of alveolar macrophages are apparent in the alveolar lumen: $\mathrm{HE} \times 99$ (original magnification).

clusters of alveolar macrophages in the alveolar lumen (Fig. 3). A lymphocyte stimulation test (DLST) was performed by evaluating the incorporation of $\mathrm{H}_{3}$-thymidine. Stimulation of pranlukast produced a value 2.3 times that of the control on day 150.

Pranlukast was discontinued on day 55, because it was presumed that the acute case of interstitial pneumonitis was induced by the drug. The patient became afebrile, and her dyspnea improved markedly 3 days after the discontinuation of the pranlukast. A chest radiograph taken at that time showed a decrease in reticulonodular infiltrates, and blood gas analysis showed improvement. At about day 120 after discontinuation, we restarted prednisolone ( $40 \mathrm{mg} / \mathrm{day}$ ) for the remaining reticulonodular infiltrates, and this resulted in a striking improvement (Fig. 1). Prednisolone was discontinued 1 month later. A chest radiograph taken 5 months after the discontinuation of prednisolone showed no abnormality.

\section{Discussion}

In drug-induced lung diseases, roentogenographic findings representing bilateral diffuse reticular or fine nodular infiltrates are usually nonspecific as to the type of interstitial pneumonitis or fibrosis. Also, the pathology with various degrees of fibrosis and mononuclear cell infiltration can be supportive, but not pathognomonic, in this entity.

Therefore, the diagnosis of drug-induced pulmonary toxicity always rests upon the exclusion of such entities as eosinophilic pneumonia, allergic broncho-pulmonary aspergillosis, Churg-Strauss syndrome, viral infection, collagen diseases and bronchiolitis obliterans-organizing pneumonia (BOOP). Pulmonary eosinophilia characterized in eosinophilic pneumonia or central bronchiectasis in allergic bronchopulmonary aspergillosis was not observed in the present case. Churg-Strauss syndome is characterized by hypereosinophilia, vasculitis, and neurologic symptoms; these were absent in this case. The patient was seronegative for cytomegalovirus, anti-nuclear and anti-DNA antibodies, and rheumatoid factor. In addition, $\mathrm{CH} 50$, C 3 and C4 complement proteins were normal. The TBLB specimen from our patient showed thickening of the alveolar septa without evidence of organization, which is incompatible with BOOP.

Interstitial lung disease is the most common and well-recognized form of pulmonary toxicity. In most cases, the mainstay therapy consists of drug withdrawal; systemic corticosteroids are administered if gas exchange abnormalities are severe.

Unfortunately, the present patient did not give consent for a challenge test. The following observations led us to diagnose the present case as an acute interstitial pneumonia induced by pranlukast ( $450 \mathrm{mg} /$ day): 1 ) acute systemic illness with prominent pulmonary alterations and fever developed after the administration of pranlukast. 2) the lymphocyte stimulation test revealed a significant positive response to pranlukast. 3) the patient's rapid improvement followed withdrawal of pranlukast. and 4) TBLB findings were consistent with drug-induced interstitial pneumonitis. Other causes, mainly infectious or environmental, were excluded.

A few reports have listed pulmonary complications in association with leukotriene receptor antagonists including pranlukast, zafirlukast, and montelukast. Zafirlukast is the first medication in this class to be approved as an $\mathrm{LTD}_{4}$-receptor antagonist, and it has been associated with such adverse effects as pulmonary infiltrates, eosinophilia and cardiomyopathy (7) and with Churg-Strauss syndrome (3). Pranlukast, too has been reported in association with Churg-Strauss syndrome (8). To our knowledge, this is the first report of an acute case of interstitial pneumonitis induced by pranlukast, it was diagnosed after discontinuation of systemic corticosteroid. Pranlukast may 


\section{TAKAHASHI et al}

need to be added to the list of agents that can cause drug-induced interstitial pneumonitis.

\section{References}

1) Barnes NC. Effects of antileukotrienes in the treatment of asthma. Am J Respir Crit Care Med 161: S73-S76, 2000.

2) Drazen JM, Israel E, O'Byrne PM. Treatment of asthma with drugs modifying the leukotriene pathway. N Engl J Med 340: 197-206, 1999.

3) Knoell DL, Lucas J, Allen JN. Churg-Strauss syndrome associated with zafirlukast. Chest 114: 332-334, 1998.

4) Miyaguchi S, Oda M, Saito H, Ishii H. Novel therapeutic approach to primary biliary cirrhosis patients, anti-eosinophil strategy. Hepatogas- troenterology 45: 1457-1461, 1998.

5) Kroegel C, Reißig A, Hengst U, Petrovic A, Häfner D, Grahmann RP. Ulcerative colitis following introduction of zafirlukast and corticosteroid withdrawal in severe atopic asthma. Eur Respir J 14: 242-243, 1999.

6) Iwanaga T, Takahashi N, Inuzuka S, Ikeda T, Hirose T, Nishima S. A case of hypereosinophilic syndrome with transient pulmonary edema following bronchoalveolar lavage. J Jpn Soc Bronchol 21: 44-48, 1999 (in Japanese, Abstract in English).

7) Wechsler ME, Garpestad E, Flier SR, et al. Pulmonary infiltrates, eosinophilia, and cardiomyopathy following corticosteroid withdrawal in patients with asthma receiving zafirlukast. JAMA 279: 455-457, 1998.

8) Kinoshita M, Shiraishi T, Koga T, Ayabe M, Rikimaru T, Oizumi K. ChurgStrauss syndrome after corticosteroid withdrawal in an asthmatic patient treated with pranlukast. J Allergy Clin Immunol 103: 534-535, 1999. 